\title{
Modelling an Orientation System based on Speculative Computation
}

\author{
João Ramos, Ken Satoh, Paulo Novais and José Neves
}

\begin{abstract}
Progress is inherent to a living society, which may occur in several different areas (e.g. computation, healthcare) and manners. The present (or now) is the time that is associated with the events perceived directly and in the first time, making, for example, the society to be very inquisitive on assistive technologies and how they may improve the human beings quality of living. This application of scientific knowledge for practical purposes may help the user in his/her diminished capabilities, and, usually, implies a small adaptation on the part of the individual in the use of devices; indeed one of the die down potentials of people with cognitive disabilities is the one of spatial orientation. On the other hand several were the authors that have developed systems to help an human being to travel between two locations. However, once the system is set up the change in the configurations have to be done manually in order to better adjust the system to the user. In order to go round this drawback, in this work it is presented a framework of speculative computation to set up the computation of the next user step using default values. When the information is obtained the computation is revised. Thus, the system may have a faster reaction to the user stimulus or it may start warning the user before he/she takes the wrong direction.
\end{abstract}

Key words: Cognitive Disabilities, Mobile Application, Guidance, Person Tracking, Ambient Intelligence, Logic Programming.

João Ramos, Paulo Novais, José Neves

Informatics Department, University of Minho, Portugal, e-mail: \{jramos,pjon,jneves\}@di.uminho.pt

Ken Satoh

National Institute of Informatics, Hitotsubashi, Chyoda-ku, Tokyo, Japan, e-mail: ksatoh@nii.ac.jp 


\section{Introduction}

The degree of assistive technology was first done in the United States in 1988 by the Technology-Related Assistance for Individuals with Disabilities Act (also named Tech Act), which was later extended in 1998 by the Assistive Technology Act $[15,1]$. The definition stated that assistive technology is any item, piece of equipment, or product system ... that is used to increase, maintain, or improve functional capabilities of individuals with disabilities. Since 1988 the interest and attention on this kind of technology has grown, leading to the development of a new set of devices to be embedded in the home environment in order to assist the user, monitoring his/her movements and his/her health on a day basis [16].

This new home environment (smarthouse [13]) prevented or minimized the independence loss on the part of the user. Caregivers and physicians may have remote access to the information collected from the environment [3], which may lead paid or not paid helpers to let the user (e.g., elder or person with cognitive disabilities) to stay in his/her home for a long time, and in an independent way.

Despite the life quality improvement that smarthouses brought, the user could only be monitored when he/she was inside the house. If the user is prevented to go outside by the caregiver he/she may be a prisoner of his/her own house, and may not have an active participation in the community.

To improve the user independency outside the premisses, researchers have been developing new ways to use the available technology and assist the user, helping $\mathrm{him} /$ her to travel alone among different locations. Since this technology has to be carried out by the user, new devices have to be small, lightweight and resistant [6, 5].On the other hand, besides the physical features of the devices, the user interface plays an important role in the utilization and acceptance of the device by the user. Usability challenges are research topics, mainly when the interfaces are developed for people with cognitive disabilities (e.g. in $[6,5,8]$ the authors concluded that simple interfaces without distractive elements and simple text are some features that should be considered when developing an application to people with cognitive disabilities).

Considering the lack of spatial orientation of the user (previously described), it was developed a system (described in [12]) that guides the user among different locations using simple interfaces, enabling the caregiver to know his/her current location, which increases either the freedom of the user or the caregiver. The former may travel alone while the latter may be engaged in another work without neglect the care provision.

In Section 2 is presented some related work developed by other authors, considering orientation systems for people with cognitive disabilities. The developed systems are intended to be executed in smartphones. Section 3 brings about the new improvements of the system developed in [12]. In Section 4 it is formulated the framework of speculative computation. Finally, in Section 5 a reflection about this work is outlined. 


\section{Related Work}

The technological development enable almost every one to have smartphones with high processing power, which may run applications that may help the owners to meet their expectations (e.g. the incorporation of a GPS module in a smartphone made possible the development of navigation programs). The major problem with these applications is that they have been developed to target the market as a whole, so that may not be used by people with disabilities, i.e., to enable people with cognitive disabilities to travel alone using a navigation system, the applications must be developed according to the client features.

Carmien et al. [2] developed a system that enables the consumer to travel among different locations using a public transportation system like the bus; indeed, they conceive an end-user programming tool that enable caregivers to create scripts according to the activities that must be carried out by the person with cognitive disabilities (physical and/or cognitive).

Liu et al. [10], picture a prototype for guiding the user through static illustrations with overlaid arrows, audio messages and text messages. The authors studied the applicability of the prototype with several real case situations (e.g. it was studied the usability of landmarks in orientation and it was concluded that a near landmark should be used instead of one outside the user's view).

AlzNav from Fraunhover Portugal [7] is an orientation system for elderly people. The orientation process is given by an arrow that acts like a compass and indicates the travel path. This system also provides a localization feature that allows caregivers to know the current user location.

The previous systems/prototypes could successfully guide the user between two locations, improving the user independence since he/she could go out alone without getting lost.

In [12] we presented a functional prototype that works as an orientation and a localization system. The user is guided through an application for Android Operative System using augmented reality, being also given to the caregivers the possibility to know the current user position in real time.

In order to enhance the guiding experience attained so far, it was conceived a Framework for Speculative Computation (presented in Section 4) to be used in these situations. As expected, using speculative computation and default values, the user may use the system to generate possible scenarios for the next step forward before it materializes, i.e., it may also be seen as an alert system. Due to the addition of this new feature, the system initially developed in [12] suffered some modifications. Section 3 describes, in brief, the changes that were done to the system.

\section{System Description}

In [11] was brought to light an initial description of the system developed in [12]. Using such a prototype the user (i.e. a person with mild cognitive disabilities) could 


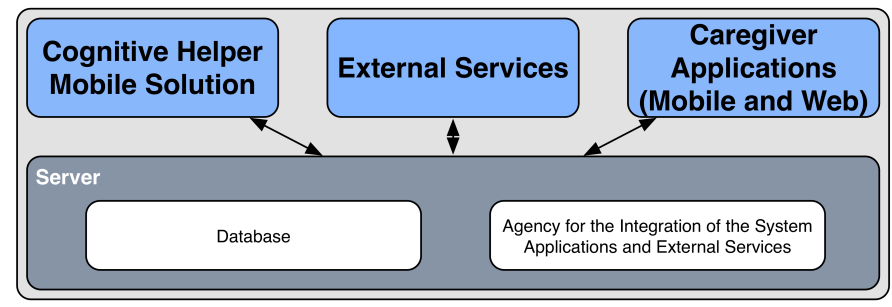

Fig. 1 An intelligible system topology

travel alone without getting lost. The system topology, i.e., the geometric properties and spatial relations are made up of (Figure 1) the server, a mobile application for people with cognitive disabilities (Cognitive Helper Mobile Solution), caregivers applications (Caregiver Applications - Mobile and Web), and external services.

The server is the system core and is made up the Database module, that stands for itself, and the agency for integration of the system applications module, that ensures the communication between the server and the user applications. To extend the features of the system, there is the possibility to connect it to external services through the agency for integration of the system applications module, i.e., there is a possibility to interact with other systems like iGenda [4].

To improve the system features it was introduced a new functionality based on speculative computation (see Section 4). This practicality is included in the mobile application for people with cognitive disabilities under the Localization Layer (Figure 2), since it needs information about the current user location, which is given by the GPS device or by the network. Using this information one may compute the location to where the user should tour.

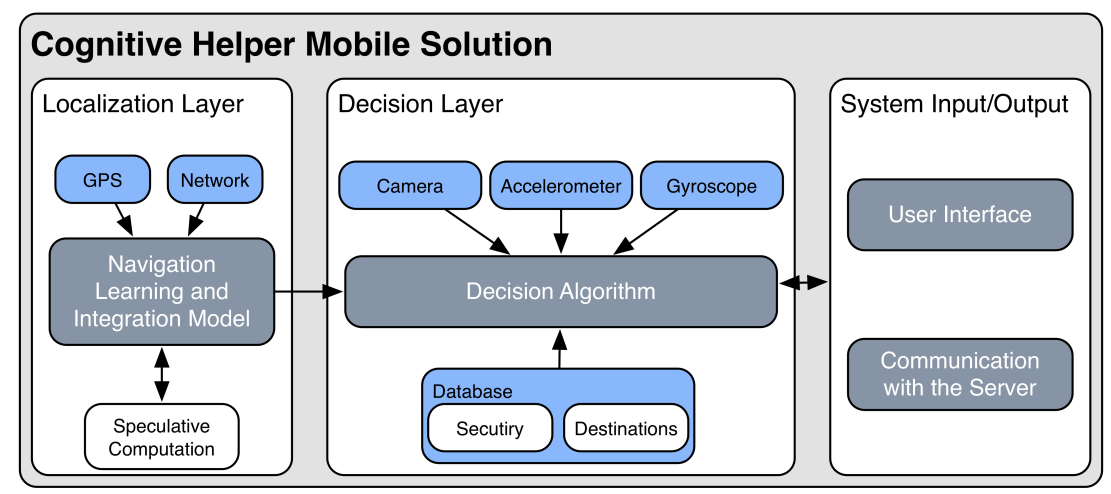

Fig. 2 Detailed framework of the mobile application for people with cognitive disabilities 


\section{A Speculative System for Users with Cognitive Disabilities}

Satoh [14], presented the theory of Speculative Computation and Abduction, which was an extension of the procedure of Kakas et al. [9]. This thesis lets the computation continue when one is faced with incomplete information, generating a tentative solution to the problem, as a result of the computational process, i.e., the missing data is complemented through a default value and, as soon as the real one is obtained, the computational process is re-examined.

A Framework of Speculative Computation in the Orientation Method for people with cognitive disabilities $\left(S F_{O M}\right)$ is now defined in terms of the signature $\left\langle\sum, \varepsilon, \Delta, \mathscr{A}, \mathscr{P}, \mathscr{I}\right\rangle[14]$, where:

- $\quad \sum$ stands for a finite set of constants (an element of $\sum$ is called a system module);

- $\varepsilon$ denotes a set of predicates called external predicates. When $Q$ is a literal belonging to an external predicate and $S$ is the identifier of the information source, $Q @ S$ is called an askable literal. We define $\sim(Q @ S)$ as $(\sim Q) @ S$;

- $\Delta$ designates the default answer set, which is a set of ground askable literals that satisfy the condition: $\Delta$ does not contain both $p\left(t_{1}, \ldots, t_{n}\right) @ S$ and $\sim p\left(t_{1}, \ldots, t_{n}\right) @ S$ at once;

- $\mathscr{A}$ is a mark of a set of predicates called abducible predicates. $Q$ is called $a b$ ducible when it is a literal with an abducible predicate;

- $\mathscr{P}$ signals a set of rules of the form:

$\triangleright H \leftarrow B_{1}, B_{2}, \ldots, B_{n}$ where $H$ is a positive ordinary literal, where each of $B_{1}, \ldots, B_{n}$ is an ordinary literal, an askable literal or an abducible; and

$\triangleright H$ is the head of rule $R$ and is named as head(R) (always non-empty), being $R$ the rule of the form $H \leftarrow B_{1}, \ldots, B_{n} ; B_{1}, \ldots, B_{n}$ is the body denoted by $\operatorname{bod} y(R)$, that in some situations is substituted by the boolean value true.

- $\mathscr{I}$ is a set of integrity constraints of the form:

$\triangleright \perp \leftarrow B_{1}, B_{2}, \ldots, B_{n}$, where $\perp$ is a contradiction special symbol and $B_{1}, B_{2}, \ldots, B_{n}$ is an ordinary literal or an askable literal or an abducible. However at least one of $B_{1}, B_{2}, \ldots, B_{n}$ is an askable literal or an abducible.

An askable literal may have different meanings, namely:

1. An askable literal $Q @ S$ in a rule $\mathscr{P}$ stands for a question put to a system module $S$ by OM; and

2. An askable literal in $\Delta$ denotes a default truth value, either true or false,i.e., $p\left(t_{1}, \ldots, t_{n}\right) @ S \in \Delta, p\left(t_{1}, \ldots, t_{n}\right) @ S$ is usually true for a question to a system module $S$, and $\sim p\left(t_{1}, \ldots, t_{n}\right) @ S \in \Delta, p\left(t_{1}, \ldots, t_{n}\right) @ S$ is generally false for a question to a system module $S$.

In the logic program given below path $(a, b)$ denotes that it is possible to travel between location $a$ and location $b$; show_next_point states that the system must show the next location (which may be an intermediate or the final location) to the user; 
show_user_warning indicates that the system must alert the user, once he/she is going in the wrong direction; and the default values for the travel path of the user are defined in $\Delta$. user_travel $(a, b)$, says that the user will travel from location $a$ to location $b$; included $(a)$ indicates if a location $a$ is part of the route.

To ensure that the user is travelling in the correct path and he/she is alerted if is out the path, a framework to Speculative Computation is given below, in terms of the logic programming suite:

$\triangleright \Sigma=\{$ gps_sensor, recognizer $\}$

$\triangleright \varepsilon=\{$ user_travel, included $\}$

$\triangleright \Delta=\{$ user_travel $(1,2) @$ gps_sensor, user_travel $(2,6) @$ gps_sensor, user_travel( 2,4$) @$ gps_sensor, user_travel( 4,5$) @$ gps_sensor, user_travel(2,3)@gps_sensor, user_travel( 3,4$) @$ gps_sensor user_travel(6,2)@gps_sensor, included(1)@ recognizer included(2)@ recognizer, included(3)@ recognizer included(4)@ recognizer, included(5)@ recognizer, included(6)@ recognizer $\}$

$\triangleright \mathscr{A}=\{$ show_next_point, show_user_warning $\}$

$\triangleright \mathscr{P}$ is a mark of the following set of rules:

guide $(A, A) \leftarrow$. guide $(A, B) \leftarrow$

$\operatorname{path}(A, F)$,

show_next_point $(F)$,

user_travel $(A, F) @ g p s \_s e n s o r$,

guide $(A, B) \leftarrow$ guide $(F, B)$.

$\operatorname{path}(A, F)$,

user_travel( $(A, F) @ g p s \_s e n s o r$, show_user_warning $(F)$,

$\operatorname{path}(1,2) \leftarrow$. guide $(F, B)$.

$\operatorname{path}(2,3) \leftarrow$.

$\operatorname{path}(2,4) \leftarrow$.

$\operatorname{path}(2,6) \leftarrow$.

$\operatorname{path}(3,4) \leftarrow$.

$\operatorname{path}(6,2) \leftarrow$.

$\operatorname{path}(4,5) \leftarrow$.

$\triangleright \mathscr{I}$ denotes the following set of integrity constraint or invariants:

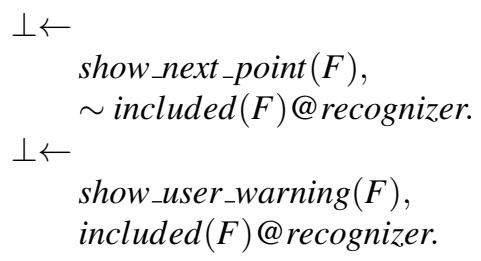


Fig. 3 Possible ways to travel among locations 1 and 5

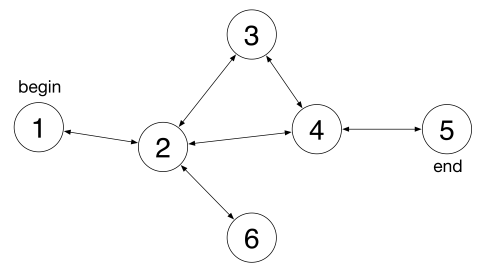

To ensure the integrity of the framework there are two invariants stating that the system may not show the next travelling point to the user if it isn't part of the route; and that the system may not alert the user if he/she is travelling in a valid path.

In the setting depicted to above it is assumed that the user will travel among locations 1 and 5 through the intermediate locations 2 and 4, and that he/she will take the wrong direction at location 2 to 6 . An elucidation of the possible paths that the user may use is presented in Figure 3.

\section{Conclusions}

The lack of orientation is a serious problem for people with cognitive disabilities. Indeed, caregivers may prevent these people to go out their homes by themselves, since the risk of getting lost may be too high. Thus, some work has been pursuit in orientation methods for people with cognitive disabilities, so they may travel alone while being remotely monitored.

In one' system the user is guided through augmented reality, surpassing the limitations of other ones that use static pictures or the explanation of a compass. On the other hand, caregivers may know in real time the current location of the user on a road map. The system may also connect to external services, thus increasing its effectiveness.

On the other hand, and to increase the system responsiveness, it has been envisage a suite for speculative computation having in mind people with cognitive disabilities.

In the upcoming work we plan to do field tests with users in order to receive their feedback and better adapt the system to their needs. The level of the prompts will be better adjust to each user, according to a speculative program of action.

Acknowledgements This work is part-funded by ERDF - European Regional Development Fund through the COMPETE Programme (operational programme for competitiveness) and by National Funds through the FCT Fundação para a Ciência e a Tecnologia (Portuguese Foundation for Science and Technology) within project FCOMP-01-0124-FEDER-028980 (PTDC/EEISII/1386/2012). The work of João Ramos is supported by a doctoral grant by FCT - Fundação para a Ciência e a Tecnologia (Portuguese Foundation for Science and Technology) SFRH/BD/89530/2012. 


\section{References}

1. Alper, S., Raharinirina, S.: Assisitive Technology for Individuals with Disabilities: A Review and Synthesis of the Literature. Journal of Special Education Technology 21(2), 47-64 (2006)

2. Carmien, S., Dawe, M., Fischer, G., Gorman, A., Kintsch, A., Sullivan, J.F.: Sociotechnical environments supporting people with cognitive disabilities using public transportation. ACM Transactions on Computer-Human Interaction 12(2), 233-262 (2005). DOI $10.1145 / 1067860.1067865$

3. Carneiro, D., Novais, P., Costa, R., Gomes, P., Neves, J., Tscheligi, M., De Ruyter, B., Markopoulus, P., Wichert, R., Mirlacher, T., Meschterjakov, A., Reitberger, W.: EMon: Embodied Monitorization. Proceedings of the European Conference on Ambient Intelligence 5859, 133-142 (2009). DOI 10.1007/978-3-642-05408-2_17

4. Costa, A., Novais, P.: An Intelligent Multi-Agent Memory Assistant. Handbook of Digital Homecare 3, 197-221 (2011)

5. Dawe, M.: Desperately seeking simplicity: how young adults with cognitive disabilities and their families adopt assistive technologies. In: Proceedings of the SIGCHI conference on Human Factors in computing systems, CHI '06, pp. 1143-1152. ACM (2006). DOI $10.1145 / 1124772.1124943$

6. Dawe, M.: Let Me Show You What I Want : Engaging Individuals with Cognitive Disabilities and their Families in Design. Technology pp. 2177-2182 (2007). DOI $10.1145 / 1240866.1240976$

7. Fraunnhover Portugal: AlzNav (2012). URL http://www.fraunhofer.pt/en/fraunhofer_aicos/projects/internal_research/alznav.html

8. Friedman, M.G., Bryen, D.N.: Web accessibility design recommendations for people with cognitive disabilities. Technology and Disability 19(4), 205-212 (2007)

9. Kakas, A.C., Kowalski, R.A., Toni, F.: The Role of Abduction in Logic Programming. Handbook of Logic in Artificial Intelligence and Logic Programming 5, 235-324 (1998)

10. Liu, A.L., Hile, H., Borriello, G., Kautz, H., Brown, P.A., Harniss, M., Johnson, K.: Informing the Design of an Automated Wayfinding System for Individuals with Cognitive Impairments. In: Proceedings of Pervasive Health '09, vol. 9, p. 8. London UK (2009)

11. Ramos, J., Anacleto, R., Costa, A., Novais, P., Figueiredo, L., Almeida, A.: Orientation System for People with Cognitive Disabilities. In: P. Novais, K. Hallenborg, D.I. Tapia, J.M.C. Rodríguez (eds.) Ambient Intelligence - Software and Applications, Advances in Intelligent and Soft Computing, vol. 153, pp. 43-50. Springer Berlin Heidelberg (2012). DOI 10.1007/978-3-642-28783-1_6

12. Ramos, J., Anacleto, R., Novais, P., Figueiredo, L., Almeida, A., Neves, J.: Geo-localization System for People with Cognitive Disabilities. In: J.B. Pérez, R. Hermoso, M.N. Moreno, J.M.C. Rodríguez, B. Hirsch, P. Mathieu, A. Campbell, M.C. Suarez-Figueroa, A. Ortega, E. Adam, E. Navarro (eds.) Trends in Practical Applications of Agents and Multiagent Systems, Advances in Intelligent Systems and Computing, vol. 221, pp. 59-66. Springer International Publishing (2013). DOI 10.1007/978-3-319-00563-8_8. URL http://dx.doi.org/10.1007/978-3-319-00563-8_8

13. Sadri, F.: Multi-Agent Ambient Intelligence for Elderly Care and Assistance. In: Aip Conference Proceedings, vol. 2007, pp. 117-120. Aip (2007). DOI 10.1063/1.3037032

14. Satoh, K.: Speculative Computation and Abduction for an Autonomous Agent. IEICE Trans. Inf. Syst. E88-D(9), 2031-2038 (2005). DOI 10.1093/ietisy/e88-d.9.2031. URL http://dx.doi.org/10.1093/ietisy/e88-d.9.2031

15. Scherer, M.M.J., Hart, T., Kirsch, N., Schulthesis, M.: Assistive Technologies for Cognitive Disabilities. Critical Reviews in Physical and Rehabilitation Medicine 17(3), 195-215 (2005). DOI 10.1615/CritRevPhysRehabilMed.v17.i3.30

16. Stefanov, D.H., Bien, Z., Bang, W.C.: The smart house for older persons and persons with physical disabilities: structure, technology arrangements, and perspectives. IEEE Transactions on Neural and Rehabilitation Systems Engineering 12(2), 228-250 (2004) 